\title{
Progressive Wave Expansions and Open Boundary Problems
}

T. Hagstrom

Institute for Computational Mechanics in Propulsion

Cleveland, Ohio

and the University of New Mexico

Albuquerque, New Mexico

S.I. Hariharan

Institute for Computational Mechanics in Propulsion

Cleveland, Ohio

and The University of Akron

Akron, Ohio

December 1995

Prepared for

Lewis Research Center

Under Cooperative Agreements NCC3-370 and NCC3-283

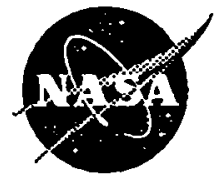

National Aeronautics and Space Administration

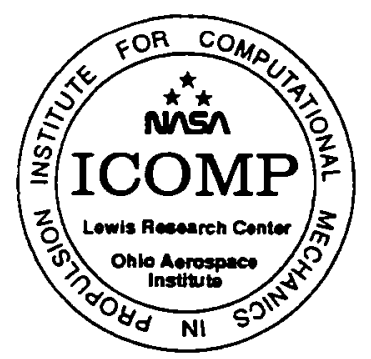




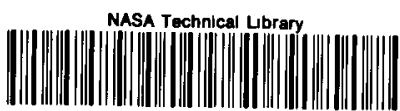

31176014237383

\title{
PROGRESSIVE WAVE EXPANSIONS AND OPEN BOUNDARY PROBLEMS \\ T HAGSTROM* AND S I HARIHARAN ${ }^{\dagger}$
}

\begin{abstract}
In this paper we construct progressive wave expansions and asymptotic boundary conditions for wave-like equations in extenor domains, including applications to electromagnetics, compressible flows and aero-acoustics The development of the conditions will be discussed in two parts The first part will include derivations of asymptotic conditions based on the well-known progressive wave expansions for the two-dimensional wave equations $A$ key feature in the denvations is that the resulting family of boundary conditions involve a single denvative in the direction normal to the open boundary These conditions are easy to implement and an application in electromagnetics will be presented The second part of the paper will discuss the theory for hyperbolic systems in two dimensions Here, the focus will be to obtain the expansions in a general way and to use them to derive a class of boundary conditions that involve only time derivatives or time and tangential denvatives Maxwell's equations and the compressible Euler equations are used as examples Simulations with the lineanzed Euler equations are presented to validate the theory
\end{abstract}

Key words. Progressive wave expansions, boundary conditions, Maxwell's Equations, Euler Equations, Numencal Simulations

AMS(MOS) subject classifications. 65M99, 35B40

1. Introduction. Exterior problems are commonly posed for wavelike equations, and their numencal solution leads to the problem of open boundary conditions. We discuss both isotropic and nonisotropic cases as they arise in electromagnetics and flud dynamics. These equations include first order hyperbolic systems such as Maxwell's equations, the Euler equations of compressible flows, or the lineanzed Euler equations, as well as second order reduced forms as approprnate Many work studies of this problem have appeared in the recent literature and we won't try to list them all There are fundamentally two different, though related, approaches that have usually been taken One is the use of high frequency asymptotics such as the geometncal optics approxamation. The other is based on the far field structure of the solution. (For a third approach based on the direct approximation of the exact condition, see [5].) Progressive wave expansions were used as a tool to construct far field boundary conditions as early as the time of Sommerfeld In the modern computational point of view, they were put in use for the first tıme by Kriegsmann and Morawetz [8] Since

* DEPARTMENT OF MATHEMATICS AND STATISTICS, THE UNIVERSITY OF NEW MEXICO, ALBUQUERQUE NM 87131, SUPPORTED IN PART BY ICOMP, NASA LEWIS RESEARCH CENTER, CLEVELAND, OH AND NSF GRANT NO DMS-9304406

† DEPARTMENT OF MATHEMATICAL SCIENCES, THE UNIVERSITY OF AKRON AKRON, OH 44325-4002, SUPPORTED IN PART BY A NASA COOPERATIVE AGREEMENT NCC - 283 AND BY ICOMP, NASA LEWIS RESEARCH CENTER, CLEVELAND, OH 
then there have been many variations to this approach. For example, extensions to anisotropic propagation were first attempted by Bayliss and Turkel [2], and a generalization to the case of anisotropic wave equations in two and three dimensions was proved by the authors [7] Issues include the construction of the expansion for general systems, their use to construct stable boundary conditions of minimum order, and, finally, their practical implementation. Higher order conditions no matter which approach is used, are typically more complicated than the partial differential equation one starts with In particular, they tend to have higher order derivatives in the direction of the propagation. To avoid this problem, often the partial differential equation itself is used. Such a procedure is not known in general for problems governed by first order hyperbolic systems. Here we provide a systematic way of dealing with this issue using progressive wave expansions. Our attention focuses on first order systems, namely, Maxwell's equations and the lnearized Euler equations To motivate the central ideas, we first consider the second order wave equation with the emphasis on progressive wave solutions.

2. Second order wave equation. As mentioned above, the goal here is to treat the problem of boundary conditions without having higher order normal denvatives. To illustrate the underlying procedure, let us consider the problem governed by the wave equation in two dimensions. We wish to construct the progressive wave solutions to this equation and exploit their structure to prescribe asymptotic boundary conditions. The equation written in cylindrical coordmates takes the form

$$
u_{t t}=u_{r r}+\frac{1}{r} u_{r}+\frac{1}{r^{2}} u_{\theta \theta}
$$

We look for solutions that are penodic in the angular direction as follows

$$
u(r, \theta, t)=\sum_{n=0}^{\infty} v_{n}(r, t) a_{n}(\theta)
$$

where $a_{n}(\theta)=A_{n} \cos n \theta+B_{n} \sin n \theta$. Substituting (2.2) in (2.1), we obtain

$$
v_{n, t t}=v_{n, r r}+\frac{1}{r} v_{n, r}-\frac{n^{2}}{r^{2}} v_{n}
$$

Following Friedlander [4], we construct solutions of (2.3) in the form

$$
v_{n}(r, t)=\sum_{j=0}^{\infty} \frac{f_{j}^{n}(t-r)}{r^{j+\frac{1}{2}}}
$$

Substitution of (2.4) in (2 3) results in the following recurrence relations

$$
f_{\jmath+1}^{n^{\prime}}(t-r)=-\frac{\left(\jmath+\frac{1}{2}\right)^{2}-n^{2}}{2(\jmath+1)} f_{\jmath}^{n}(t-r)
$$


The goal is to examine the effect of the recurrence relations on constructions of asymptotic boundary conditions. First, we observe that substitution of (2.4) in (2.2) yeelds the following formal representation of the solution

$$
u(r, \theta, t)=\sum_{n=0}^{\infty} a_{n}(\theta) \sum_{j=0}^{\infty} \frac{f_{j}^{n}(t-r)}{r^{j+\frac{1}{2}}}
$$

Manipulations of this senes, particularly increasing the order of the decay rate for boundary conditions, have been proposed by many authors (e.g. Bayliss and Turkel [1]). In fact, a different form of (26) has been used for these constructions, which will not be discussed here. We define a "basic boundary operator" from (2.6) as follows:

$$
B=\frac{\partial}{\partial t}+\frac{\partial}{\partial r}+\frac{1}{2 r}
$$

It is immediately verified from (26) that

$$
B u=-\sum_{n=0}^{\infty} a_{n}(\theta) \sum_{j=1}^{\infty} \frac{\jmath f_{j}^{n}(t-r)}{r^{j+\frac{3}{2}}}
$$

Direct approximation of $(28)$ is the radiation condition $B u=0$, a popular condition in the literature noted by many researchers (e g. Bayliss and Turkel [1], Engquist and Majda [3]). Asymptotic accuracy of such a condition is $0\left(r^{-5 / 2}\right)$, which is evident from (2.8). Hugher order conditions in general require higher order normal derivatives or denvatives in the dominant direction of propagation This may not be a desirable feature numerically, particularly for nonlinear generallzations. Here we obtain higher order conditions that involve $B u, u, u_{\theta \theta}$, and their time derivatives on the artificial boundary. We begin with the construction of higher order conditions by differentiating $(28)$. This yields:

$$
\frac{\partial}{\partial t} B u=-\sum_{n=0}^{\infty} a_{n}(\theta) \sum_{j=0}^{\infty} \frac{\jmath f_{j}^{n^{\prime}}(t-r)}{r^{j+\frac{3}{2}}}
$$

Noting that the inner summation may be written in the form

$$
\sum_{j=0}^{\infty} \frac{(\jmath+1) f_{j+1}^{n^{\prime}}(t-r)}{r^{\jmath+\frac{5}{2}}}
$$

and using the recurrence relation $(25)$ yrelds:

$$
\frac{\partial}{\partial t} B u=\sum_{n=0}^{\infty} a_{n}(\theta) \sum_{\jmath=0}^{\infty} \frac{\left(\jmath+\frac{1}{2}\right)^{2}-n^{2}}{2 r^{j+\frac{3}{2}}} f_{\jmath}^{n}
$$


A simple manipulation of the nght hand side yields:

$$
\frac{\partial}{\partial t} B u-\frac{1}{8 r^{2}} u-\frac{1}{2 r^{2}} \frac{\partial^{2} u}{\partial \theta^{2}}=\frac{1}{2} \sum_{n=0}^{\infty} a_{n}(\theta) \sum_{j=1}^{\infty} \frac{\jmath(\jmath+1)}{r^{\jmath+\frac{3}{2}}} f_{j}^{n}
$$

The highlight here is the observation

$$
u_{\theta \theta}=-\sum_{n=0}^{\infty} n^{2} a_{n}(\theta) \sum_{j=0}^{\infty} \frac{f_{j}^{n}(t-r)}{r^{j+\frac{1}{2}}}
$$

Note that the asymptotic accuracy of the candidate boundary condition (2 10) is increased further to $0\left(r^{-\frac{7}{2}}\right)$ Let

$$
B_{1} u=\frac{\partial}{\partial t} B u-\frac{u}{8 r^{2}}-\frac{u_{\theta \theta}}{r^{2}}
$$

Then (2 10) becomes

$$
B_{1} u=\frac{1}{2} \sum_{n=0}^{\infty} a_{n}(\theta) \sum_{\jmath=0}^{\infty} \frac{(\jmath+1)(\jmath+2)}{r^{\jmath+\frac{7}{2}}} f_{\jmath+1}^{n}
$$

This form again suggests the use of the recurrence relations $(25)$ by differentiating the equation with respect to time Doing so, we obtain

$$
\frac{\partial}{\partial t} B_{1} u=-\frac{1}{4} \sum_{n=0}^{\infty} a_{n}(\theta) \sum_{j=0}^{\infty} \frac{(\jmath+2)\left[\left(\jmath+\frac{1}{2}\right)^{2}-n^{2}\right]}{r^{j+\frac{7}{2}}} f_{\jmath}^{n}
$$

which is equivalent to

$$
\text { (2.15) } \frac{\partial}{\partial t} B_{1} u+\frac{1}{8 r^{3}} u+\frac{1}{2 r^{3}} u_{\theta \theta}=-\frac{1}{4} \sum_{n=0}^{\infty} a_{n}(\theta) \sum_{\jmath=1}^{\infty} \frac{\jmath\left[\left(\jmath+\frac{3}{2}\right)^{2}-n^{2}\right]}{r^{\jmath+\frac{7}{2}}} f_{\jmath}^{n}
$$

We note that $-n^{2}$ translates into the second tangential derivative Defining

$$
B_{2} u=\frac{\partial}{\partial t} B_{1} u+\frac{1}{8 r^{3}} u+\frac{1}{2 r^{3}} u_{\theta \theta}
$$

it is clear that equation (2.15) yields a one asymptotic order higher boundary condition (to $O\left(r^{-\frac{9}{2}}\right)$ ). Moreover, noting

$$
B_{2} u=-\frac{1}{4} \sum_{n=0}^{\infty} a_{n}(\theta) \sum_{\jmath=0}^{\infty} \frac{(\jmath+1)\left[\left(\jmath+\frac{5}{2}\right)^{2}-n^{2}\right]}{r^{j+\frac{s}{2}}} f_{\jmath+1}^{n}
$$

and applying the time derivative again, the process becomes clear and it yıelds

$$
B_{3} u=\frac{\partial}{\partial t} B_{2} u-\frac{25}{128 r^{4}} u-\frac{13}{16 r^{4}} u_{\theta \theta}-\frac{1}{8 r^{4}} u_{\theta \theta \theta \theta}=O\left(\frac{1}{r^{\frac{11}{2}}}\right)
$$


Remark 1: As far as numencal implementation of these conditions are concerned, one may consider a sequence of operations to update $u$ at the current tıme:

$$
\begin{gathered}
u_{t}+u_{r}+\frac{1}{2 r} u=z \\
z_{t}-\frac{1}{8 r^{2}} u+\frac{1}{r^{2}} u_{\theta \theta}=v \\
v_{t}+\frac{1}{8 r^{3}} u+\frac{1}{2 r^{3}} u_{\theta \theta}=w \\
w_{t}-\frac{25}{128 r^{4}} u-\frac{13}{16 r^{4}} u_{\theta \theta}-\frac{1}{8 r^{4}} u_{\theta \theta \theta \theta}=0
\end{gathered}
$$

The above sequence of equations (which provides a boundary condition asymptotically accurate to $\left.O\left(r^{-\frac{11}{2}}\right)\right)$ as a system of first order equations to march in time. (At the time this article was written one of the students of the second author has implemented such a procedure and obtained the indicated asymptotic improvement. The details will appear elsewhere).

Remark 2: The procedure above coincides with the high frequency approximations of the exact condition in the radially symmetric case In the Laplace transform domain, the exact operator has the form (see [6])

$$
B_{e} u=-\frac{1}{r} \frac{s r K_{0}^{\prime}(s r)}{K_{0}(s r)} .
$$

Where $K_{0}(z)$ is the modified Bessel function of order 0 . Moreover we find as $s r \rightarrow \infty$

$$
B_{e} u=\frac{1}{r}\left(s r+\frac{1}{2}-\frac{1}{8 s r}+\frac{1}{8(s r)^{2}}+O\left((s r)^{-3}\right)\right)
$$

The Laplace transform of the derived operators coincides with the large $s r$ approximations of the exact boundary operator $B_{e}$ We can, then, interpret the expansions both as a long-range and as a high-frequency approximation.

We also note that the Fourier transform of the operator $B_{1} u$ coincides with the second order operator proposed by Kriegsmann et al. [9] in conjunction with on surface radiation conditions. As an example we consider the computation of the surface current calculation in electromagnetic scattering. Let $\Gamma$ be the boundary of a perfect conductor Then the magnitude of the total current is given by the formula (see [9])

$$
J=\left|\frac{2}{k} \frac{\partial}{\partial n}\left(u_{s}+u_{\imath n c}\right)\right|_{\Gamma}
$$


where $u_{s}$ is the scattered field, and $u_{2 n c}$ is the known incident field For perfect conductors $u_{s}=-u_{3 n c}$ on the boundary of the scatterer $\Gamma$ The principle of the on surface boundary procedure consists of bringing the far field boundary exactly on the interface of the scatterer The advantage is rather clear. Since the total current is a functional of the normal derivative of the scattered field and the radiation boundary operators on the surface directly express the normal derivatives in terms of the incident field We note that in the formula for the surface current $k$ is the wave number which arose from the Fourier transform of the wave equation We list the Founer transform of the operators derived in our theory They are:

Condition 1

$$
-\imath k u+u_{r}+\frac{1}{2 r} u=0
$$

Condition 2.

$$
-\imath k\left(-\imath k u+u_{r}+\frac{1}{2 \tau} u\right)=\frac{1}{8 r^{2}}+\frac{1}{2 r^{2}} u_{\theta \theta}
$$

Condition 3.

$(2.28)-2 k)^{2}\left(-\imath k u+u_{\tau}+\frac{1}{2 \tau} u\right)=-\imath k\left(\frac{1}{8 r^{2}} u+\frac{1}{2 r^{2}} u_{\theta \theta}\right)-\frac{1}{8 r^{3}} u-\frac{1}{2 \tau^{3}} u_{\theta \theta}$

The first two operators are used in [9], and the third one is, so far as we know, new A plane wave incident upon a unit cylinder is considered for the calculation of $J$ and results are shown in Figures $2.1(k=5)$ and 22 $(k=2)$ respectively. The incident field has the specific form $u_{2 n c}=e^{2 k r \cos \theta}$

Remark 3.: For anisotropic equations, such as convective wave equation, an analogous procedure may be derived. The use of the resulting conditions are more pertinent to systems of equations such as the lineanzed Euler equation This is discussed in section 4 . 


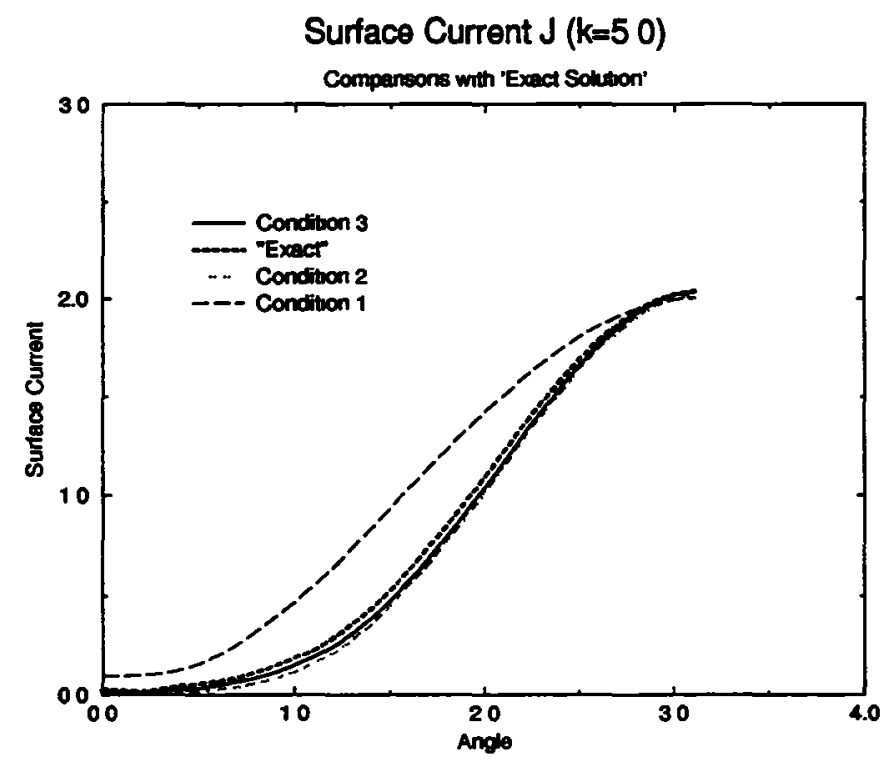

Fig 21 Comparzson of results wrth exact solution, $k=5$

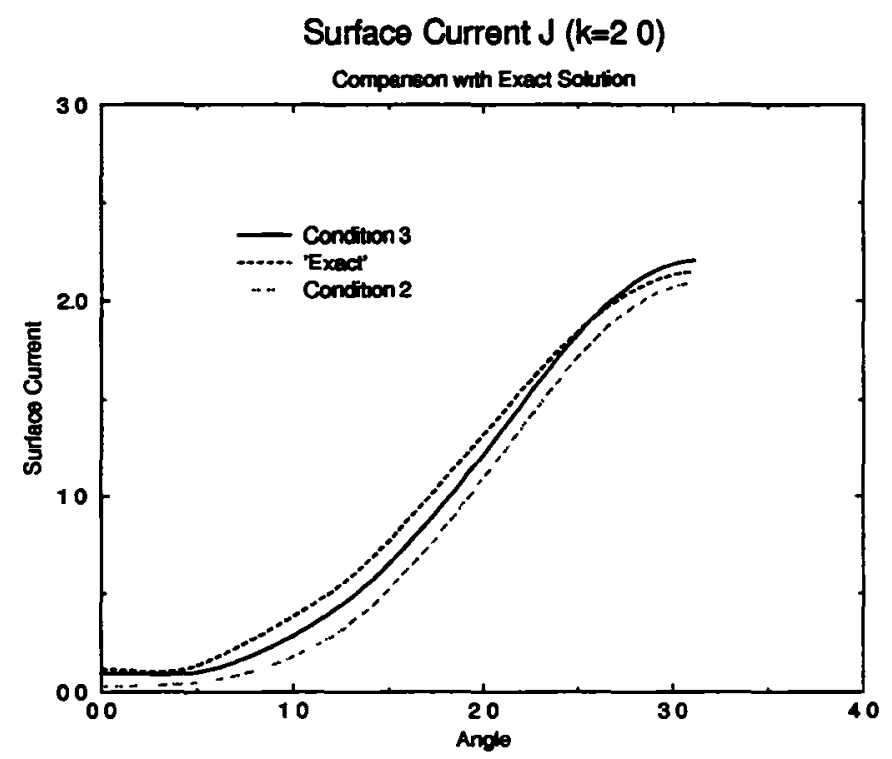

FIG 22 Comparzson of results wrth exact solution, $k=2$ 
3. First order hyperbolic systems - Isotropic case. Here our focus is to extend the ideas to systems of first order equations The progressive wave expansions may be carried out directly in the time domain as we did for the second order wave equations or in the Laplace transform domain. In this section we present the construction using the Laplace transform. The direct approach is illustrated in Section 4.

Maxwell's equations offer an interesting example of an isotropic system Here we confine our attention to Transverse Magnetic (TM) fields for simplicity. The full field equations are:

$$
\begin{gathered}
d \imath v \in \mathbf{E}=d \imath v \mu \mathbf{H}=0, \\
\text { curl } \mathbf{H}=\epsilon \frac{\partial \mathrm{E}}{\partial t}, \\
\text { curl } \mathbf{E}=-\mu \frac{\partial \mathbf{H}}{\partial t}
\end{gathered}
$$

We shall consider TM fields as follows:

$$
\mathbf{E}=E(x, y, t) \mathbf{k}
$$

Equation (3.4) indicates that the electric field propagates in the direction perpendicular to the $x-y$ plane and is transverse to the magnetic field Under these assumptions, equations (3.2) and (3.3) become

$$
\frac{\partial H_{2}}{\partial x}-\frac{\partial H_{1}}{\partial y}=\epsilon \frac{\partial E}{\partial t}
$$

and

$$
\frac{\partial E}{\partial y} \mathbf{i}-\frac{\partial E}{\partial x} \mathbf{j}=-\mu\left(\frac{\partial H_{1}}{\partial t} \mathbf{i}+\frac{\partial H_{2}}{\partial t} \mathbf{j}\right)
$$

respectively Rearranging equations (3 6) and (3 7), we obtain the following system

$$
\begin{gathered}
\frac{\partial E}{\partial t}=\frac{1}{\epsilon}\left[\frac{\partial H_{2}}{\partial x}-\frac{\partial H_{1}}{\partial y}\right] \\
\frac{\partial H_{1}}{\partial t}=-\frac{1}{\mu} \frac{\partial E}{\partial y} \\
\frac{\partial H_{2}}{\partial t}=\frac{1}{\mu} \frac{\partial E}{\partial x}
\end{gathered}
$$


This can be put in the conventional form:

$$
\mathbf{u}_{t}=\mathbf{A} \mathbf{u}_{x}+\mathbf{B} \mathbf{u}_{y}
$$

where

$$
\begin{gathered}
\mathbf{A}=\left(\begin{array}{ccc}
0 & 0 & \frac{1}{\epsilon} \\
0 & 0 & 0 \\
\frac{1}{\mu} & 0 & 0
\end{array}\right), \\
\mathbf{B}=\left(\begin{array}{ccc}
0 & -\frac{1}{\epsilon} & 0 \\
-\frac{1}{\mu} & 0 & 0 \\
0 & 0 & 0
\end{array}\right)
\end{gathered}
$$

and where $\mathrm{u}=\left(E, H_{1}, H_{2}\right)^{T}$ Converting to polar coordnates we obtain.

$$
\begin{aligned}
\frac{\partial}{\partial t}\left(\begin{array}{c}
E \\
H_{1} \\
H_{2}
\end{array}\right) & =\left(\begin{array}{ccc}
0 & -\frac{1}{\epsilon} \sin \theta & \frac{1}{\epsilon} \cos \theta \\
-\frac{1}{\mu} \sin \theta & 0 & 0 \\
\frac{1}{\mu} \cos \theta & 0 & 0
\end{array}\right) \frac{\partial}{\partial r}\left(\begin{array}{c}
E \\
H_{1} \\
H_{2}
\end{array}\right) \\
& +\frac{1}{\tau}\left(\begin{array}{ccc}
0 & -\frac{1}{\epsilon} \cos \theta & -\frac{1}{\epsilon} \sin \theta \\
-\frac{1}{\mu} \cos \theta & 0 & 0 \\
-\frac{1}{\mu} \sin \theta & 0 & 0
\end{array}\right) \frac{\partial}{\partial \theta}\left(\begin{array}{c}
E \\
H_{1} \\
H_{2}
\end{array}\right)
\end{aligned}
$$

We take the Laplace transform of (3.9). With the change of variable $\tilde{r}=r s$ we have

$$
\left(\begin{array}{c}
\hat{E} \\
\hat{H}_{1} \\
\hat{H}_{2}
\end{array}\right)=\left(R \frac{\partial}{\partial \hat{r}}+\frac{1}{\bar{r}} \Theta \frac{\partial}{\partial \theta}\right)\left(\begin{array}{c}
\hat{E} \\
\hat{H}_{1} \\
\hat{H}_{2}
\end{array}\right)
$$

where

$$
R=\left(\begin{array}{ccc}
0 & -\frac{1}{\epsilon} \sin \theta & \frac{1}{\epsilon} \cos \theta \\
-\frac{1}{\mu} \sin \theta & 0 & 0 \\
\frac{1}{\mu} \cos \theta & 0 & 0
\end{array}\right)
$$

and

$$
\Theta=\left(\begin{array}{ccc}
0 & -\frac{1}{\epsilon} \cos \theta & -\frac{1}{\epsilon} \sin \theta \\
-\frac{1}{\mu} \cos \theta & 0 & 0 \\
-\frac{1}{\mu} \sin \theta & 0 & 0
\end{array}\right)
$$

We seek an expansion of solutions of (3 10) in the form

$$
\left(\begin{array}{c}
\hat{E} \\
\hat{H}_{1} \\
\hat{H}_{2}
\end{array}\right)=\frac{e^{-\dot{r} g(\theta)}}{\tilde{r}^{\alpha}}\left(\mathbf{a}+\frac{1}{\bar{r}} \mathbf{b}+\cdots\right)
$$


We note that this form is similar to Fnedlander's form that apples to the second order wave equation in the Laplace transform domain Also, we have introduced a decay rate constant $\alpha$ which turns out in two dimensions to equal $\frac{1}{2}$, as expected Substituting (3.11) into (3.10) yields to leading order:

$$
A=I+g R+g^{\prime} \Theta
$$

and

$$
A \mathbf{a}=\mathbf{0}
$$

For this requirement to be true, clearly it must follow that-

$$
\begin{aligned}
& 0=\operatorname{det}(A)=\operatorname{det}\left(\begin{array}{ccc}
1 & -\frac{1}{\epsilon}\left(g \sin \theta+g^{\prime} \cos \theta\right) & \frac{1}{\epsilon}\left(g \cos \theta-g^{\prime} \sin \theta\right) \\
-\frac{1}{\mu}\left(g \sin \theta+g^{\prime} \cos \theta\right) & 1 & 0 \\
\frac{1}{\mu}\left(g \cos \theta-g^{\prime} \sin \theta\right) & 0 & 1
\end{array}\right) \\
& =\left(1-\frac{1}{\epsilon \mu}\left(\left(g \sin \theta+g^{\prime} \cos \theta\right)^{2}+\left(g \cos \theta-g^{\prime} \sin \theta\right)^{2}\right)\right) \\
& =1-\frac{1}{\epsilon \mu}\left(g^{2}+\left(g^{\prime}\right)^{2}\right)
\end{aligned}
$$

The roots of equation (3.14) are:

$$
g= \pm \sqrt{\epsilon \mu}, \sqrt{\epsilon \mu} \cos (\theta+\phi)
$$

$\phi$ arbitrary. For waves propagating to infinity in all directions we choose $g=\sqrt{\epsilon \mu}$ as the allowoable root With this value of $g$, the matrix $A$ becomes

$$
A=\left(\begin{array}{ccc}
1 & -\sqrt{\frac{\mu}{\epsilon}} \sin \theta & \sqrt{\frac{\mu}{\epsilon}} \cos \theta \\
-\sqrt{\frac{\varepsilon}{\mu}} \sin \theta & 1 & 0 \\
\sqrt{\frac{\epsilon}{\mu}} \cos \theta & 0 & 1
\end{array}\right)
$$

whose nght nullvector is

$$
\mathbf{a}=\left(\begin{array}{c}
1 \\
\sqrt{\frac{\epsilon}{\mu}} \sin \theta \\
-\sqrt{\frac{\epsilon}{\mu}} \cos \theta
\end{array}\right) a_{1}(\theta)
$$

with left nullvector

$$
\mathrm{l}^{T}=\left(1, \sqrt{\frac{\mu}{\epsilon}} \sin \theta,-\sqrt{\frac{\mu}{\epsilon}} \cos \theta\right)
$$


The next order terms in the asymptotic expansion yield the following relation:

$$
A \mathbf{b}=-\alpha R \mathbf{a}+\Theta \frac{\partial \mathbf{a}}{\partial \theta}
$$

and $\alpha$ is determined by.

$$
\alpha=\frac{\mathbf{l}^{T} \Theta \frac{\partial \mathrm{a}}{\partial \theta}}{\mathbf{l}^{T} R \mathbf{a}}
$$

1 e., by requring $(1, A b)=0$

Noting the following calculations:

$$
\begin{gathered}
R \mathbf{a}=a_{1}(\theta)\left(\begin{array}{c}
-\frac{1}{\sqrt{\epsilon \mu}} \\
-\frac{1}{\mu} \sin \theta \\
\frac{1}{\mu} \cos \theta
\end{array}\right) \\
\frac{\partial \mathbf{a}}{\partial \theta}=\frac{\partial a_{1}}{\partial \theta}\left(\begin{array}{c}
1 \\
\sqrt{\frac{\epsilon}{\mu}} \sin \theta \\
-\sqrt{\frac{\epsilon}{\mu}} \cos \theta
\end{array}\right)+a_{1}\left(\begin{array}{c}
0 \\
\sqrt{\frac{\epsilon}{\mu}} \cos \theta \\
\sqrt{\frac{\epsilon}{\mu}} \sin \theta
\end{array}\right), \\
\Theta \frac{\partial \mathbf{a}}{\partial \theta}=\frac{\partial a_{1}}{\partial \theta}\left(\begin{array}{c}
0 \\
-\frac{1}{\mu} \cos \theta \\
-\frac{1}{\mu} \sin \theta
\end{array}\right)+a_{1}-\left(\begin{array}{c}
-\frac{1}{\sqrt{\epsilon \mu}} \\
0 \\
0
\end{array}\right) \\
\mathbf{1}^{T} R \mathbf{a}=a_{1}(\theta)\left(\frac{-2}{\sqrt{\epsilon \mu}}\right), \mathbf{l}^{T} \Theta \frac{\partial \mathbf{a}}{\partial \theta}=a_{1}(\theta)\left(\frac{-1}{\sqrt{\epsilon \mu}}\right)
\end{gathered}
$$

it follows that

$$
\alpha=\frac{1}{2}
$$

Choose

$$
\mathbf{b}=\left(\begin{array}{c}
0 \\
b_{2} \\
b_{3}
\end{array}\right)
$$

and use the last two equations to obtain

$$
b_{2}=\left(\frac{1}{2 \mu} \sin \theta\right) a_{1}-\frac{1}{\mu} \cos \theta \frac{\partial a_{1}}{\partial \theta}
$$




$$
b_{3}=\left(-\frac{1}{2 \mu} \cos \theta\right) a_{1}-\frac{1}{\mu} \sin \theta \frac{\partial a_{1}}{\partial \theta} .
$$

Substituting $a$ and $b$ in (3.11) we obtain

$$
\begin{gathered}
\hat{E}=\frac{e^{-\tilde{r} \sqrt{\mu \epsilon}}}{\tilde{r}^{\frac{1}{2}}} a_{1}, \quad \frac{\partial \hat{E}}{\partial \theta}=\frac{e^{-\tilde{r} \sqrt{\mu \epsilon}}}{\tilde{r}^{\frac{1}{2}}} \frac{\partial a_{1}}{\partial \theta}, \\
\hat{H}_{1}=\sqrt{\frac{\epsilon}{\mu}} \sin \theta \hat{E}+\frac{1}{\mu r s}\left(\frac{\sin \theta}{2} \hat{E}-\cos \theta \frac{\partial \hat{E}}{\partial \theta}\right), \\
\hat{H}_{2}=-\sqrt{\frac{\epsilon}{\mu}} \cos \theta \hat{E}+\frac{1}{\mu r s}\left(\frac{-\cos \theta}{2} \hat{E}-\sin \theta \frac{\partial \hat{E}}{\partial \theta}\right) .
\end{gathered}
$$

Multiplying through by $s$ and taking the inverse transform finally yields

$$
\begin{gathered}
\frac{\partial H_{1}}{\partial t}=\left(\sqrt{\frac{\epsilon}{\mu}} \sin \theta \frac{\partial}{\partial t}+\frac{\sin \theta}{2 \mu r}-\frac{\cos \theta}{\mu r} \frac{\partial}{\partial \theta}\right) E \\
\frac{\partial H_{2}}{\partial t}=-\left(\sqrt{\frac{\epsilon}{\mu}} \cos \theta \frac{\partial}{\partial t}+\frac{\cos \theta}{2 \mu r}+\frac{\sin \theta}{\mu r} \frac{\partial}{\partial \theta}\right) E
\end{gathered}
$$

As only one boundary condition is required, we convert these into a single condition Multiplying (3.17) $\sin \theta$ and subtracting (3.18) multiplied by $\cos \theta$, we obtain our final form.

$$
\frac{\partial H_{1}}{\partial t} \sin \theta-\frac{\partial H_{2}}{\partial t} \cos \theta-\sqrt{\frac{\epsilon}{\mu}} \frac{\partial E}{\partial t}=\frac{1}{2 \mu r} E
$$

This construction is easly extended to higher order, though we have not devised a unified approach to the implementation of the higher order conditons.

4. The linearized Euler equations - An anisotropic example. The construction of asymptotic boundary conditions for the linearized and nonlinear compressible Euler equations is also of interest, partıcularly for applications in aeroacoustics. In this section, we construct the expansions in the time domain durectly. Agarn, the system takes the form:

$$
\mathbf{u}_{t}=\mathbf{A} \mathbf{u}_{x}+\mathbf{B} \mathbf{u}_{\mathbf{y}}
$$

where $\mathbf{A}$ and $\mathbf{B}$ are constant matrices In cylndrical coordinates we have

$$
\mathbf{u}_{t}=\mathbf{R} \mathbf{u}_{r}+\frac{1}{r} \mathbf{T} \mathbf{u}_{\theta},
$$


where

$$
\begin{aligned}
\mathbf{R} & =\mathbf{A} \cos \theta+\mathbf{B} \sin \theta \\
\mathbf{T} & =-\mathbf{A} \sin \theta+\mathbf{B} \cos \theta
\end{aligned}
$$

A far field asymptotic expansion may be constructed in the following form for the solution vector $u$ :

$$
\mathbf{u}=\sum_{n=0}^{\infty} \frac{f_{n}(t-r g(\theta))}{r^{n+\frac{1}{2}}} \mathbf{a}_{n}
$$

where the scalar function $g(\theta)$ and the vectors $a_{n}(\theta)$ are to be determined The function $f_{0}$ is analogous to the radiation function discussed in [4] The other functions are recursively determined by substitution of the expansion into equation (4.2). The $O\left(\frac{1}{r}\right)$ terms yield:

$$
\mathrm{Ca}_{0}=\mathbf{0},
$$

where $\mathbf{C}=\mathbf{I}+g(\theta) \mathbf{R}+g^{\prime}(\theta) \mathbf{T}$ For $\mathbf{a}_{0}$ to be nontrivial one must have $\operatorname{det}(\mathbf{C})=0$, yielding an 'eikonal function' $g(\theta)$. The next order correction yields

$$
f_{0} \mathbf{C a _ { 1 }}=-f_{1}^{\prime} \frac{1}{2} \mathbf{R} \mathbf{a}_{0}+\mathrm{T} \frac{\partial \mathbf{a}_{0}}{\partial \theta}
$$

This imposes a necessary restriction that $f_{0}=f_{1}^{\prime}$ In general, it follows $f_{n-1}=f_{n}^{\prime}, n \geq 1$. At this point, we turn to the isentropic, linearned, compressible, Euler equations to llustrate the actual calculations involved in solving these algebrasc problems For a uniform base flow in the $x$ direction they are-

$$
\begin{gathered}
\left(\frac{\partial}{\partial t}+M \frac{\partial}{\partial x}\right) p+\frac{\partial u}{\partial x}+\frac{\partial v}{\partial y}=0 \\
\left(\frac{\partial}{\partial t}+M \frac{\partial}{\partial x}\right) u+\frac{\partial p}{\partial x}=0 \\
\left(\frac{\partial}{\partial t}+M \frac{\partial}{\partial x}\right) v+\frac{\partial p}{\partial y}=0
\end{gathered}
$$

Conversion of this system to cylundncal coordinates $(48)-(4.10)$ takes the form (4 2) where

$$
\mathbf{u}=\left(\begin{array}{c}
p \\
u \\
v
\end{array}\right)
$$




$$
\begin{gathered}
\mathbf{R}=\left(\begin{array}{ccc}
M \cos \theta & \cos \theta & \sin \theta \\
\cos \theta & M \cos \theta & 0 \\
\sin \theta & 0 & M \cos \theta
\end{array}\right) \\
\mathbf{T}=\left(\begin{array}{ccc}
-M \sin \theta & -\sin \theta & \cos \theta \\
-\sin \theta & -M \sin \theta & 0 \\
\cos \theta & 0 & -M \sin \theta
\end{array}\right)
\end{gathered}
$$

Calculation of $g(\theta)$ for these equations yreld.

$$
g(\theta)=\frac{1}{\sqrt{1-M^{2} \sin ^{2} \theta}+M \cos \theta},
$$

and the matrix $\mathbf{C}$ has the form

$$
\mathbf{C}=\left[\begin{array}{ccc}
1-M Q & -Q & -R \\
-Q & 1-M Q & 0 \\
-R & 0 & 1-M Q
\end{array}\right]
$$

where

$$
\begin{aligned}
& Q=g \cos \theta-g^{\prime} \sin \theta, \\
& R=g \sin \theta+g^{\prime} \cos \theta
\end{aligned}
$$

Solutions of (4.6) are given by

$$
\mathbf{a}_{0}=h_{0}(\theta)\left(\begin{array}{c}
\frac{1}{Q} \\
\frac{1-M Q}{R} \\
\frac{1-M Q}{1-M Q}
\end{array}\right)=h_{0}(\theta)\left(\begin{array}{c}
1 \\
\tau_{2} \\
r_{3}
\end{array}\right),
$$

and the solutions of (47) are given by:

$$
\mathbf{a}_{1}=h_{1}(\theta)\left(\begin{array}{c}
1 \\
r_{2} \\
r_{3}
\end{array}\right)+h_{0}(\theta)\left(\begin{array}{c}
0 \\
b_{2} \\
b_{3}
\end{array}\right)+h_{0}^{\prime}(\theta)\left(\begin{array}{c}
0 \\
c_{2} \\
c_{3}
\end{array}\right)
$$

Here $h_{0}(\theta)$ and $h_{1}(\theta)$ are arbitrary functions of $\theta$ and the coefficients $b_{\imath}$ and $c_{2}$ are given by

$$
\begin{aligned}
& b_{2}=\frac{\left(\cos \theta+M r_{2} \cos \theta\right) / 2+M r_{2}^{\prime} \sin \theta}{1-M Q}, \\
& b_{3}=\frac{\left(\sin \theta+M r_{3} \cos \theta\right) / 2+M r_{3}^{\prime} \sin \theta}{1-M Q},
\end{aligned}
$$




$$
\begin{gathered}
c_{2}=\frac{\sin \theta+M r_{2} \sin \theta}{1-M Q}, \\
c_{3}=\frac{-\cos \theta+M r_{3} \sin \theta}{1-M Q} .
\end{gathered}
$$

Collection of these results in the asymptotic expansions yrelds (to $O\left(r^{-5 / 2}\right)$ ):

$$
p=\frac{h_{0} f_{0}}{r^{1 / 2}}+\frac{h_{1} f_{1}}{r^{3 / 2}}
$$

$$
u=r_{2} p+\frac{h_{0} f_{1}}{r^{3 / 2}} b_{2}+\frac{h_{0}^{\prime} f_{1}}{r^{3 / 2}} c_{2},
$$

$$
v=r_{3} p+\frac{h_{0} f_{1}}{r^{3 / 2}} b_{3}+\frac{h_{0}^{\prime} f_{1}}{r^{3 / 2}} c_{3}
$$

Differentiating $u$ and $v$ with respect to $t$ and using the result $f_{0}=f_{1}^{\prime}$ to $O\left(r^{-5 / 2}\right)$, we have

$$
\begin{aligned}
& u_{t}=r_{2} p_{t}+\frac{p}{r} b_{2}+\frac{h_{0}^{\prime} f_{0}}{r^{3 / 2}} c_{2}, \\
& v_{t}=r_{3} p_{t}+\frac{p}{\tau} r_{3}+\frac{h_{0}^{\prime} f_{0}}{r^{3 / 2}} c_{3}
\end{aligned}
$$

Finally, noting the term involving $h_{0}^{\prime}$ can be eliminated from the last two equations, we have

$$
\alpha p_{t}+\beta u_{t}+\gamma v_{t}=\frac{p}{r} \delta
$$

where

$$
\begin{gathered}
\alpha=r_{2} c_{3}-r_{3} c_{2}, \\
\beta=-c_{2}, \\
\gamma=c_{3}, \\
\delta=b_{3} c_{2}-b_{2} c_{3}
\end{gathered}
$$

The higher order conditions are obtained in a simular manner In fact, one can show that the next order condition is of the form

$$
(\alpha p+\beta u+\gamma v)_{t t}=\frac{p_{t}}{\tau} \delta+\frac{p}{r^{2}} \epsilon
$$


which is accurate to $O\left(r^{-7 / 2}\right)$. Note that these condrtions do not involve any spatial derivatives As such they are ideal for rectangular domains where typically one has to pay special attention to the corners, particularly when high order numerical schemes are used These conditions correspond to the primary acoustic boundary condition. In addition, one must impose at inflow boundaries, a vorticity condition For nonsentropic flows, in addition to the vorticity, entropy must also be specified at inflow At inflow the $y$ momentum equation and zero vorticity condition yield an exact relation

$$
v_{t}+(p+M u)_{y}=0 .
$$

5. A Model Problem. In this section we begin with the linearzed Euler equations with mean velocity convection. The scaled form of these equations are identical to the one that we used to derive the conditions, except they contain forcing terms that characterize a driving source They are.

$$
\begin{gathered}
\left(\frac{\partial}{\partial t}+M \frac{\partial}{\partial x}\right) p+\frac{\partial u}{\partial x}+\frac{\partial v}{\partial y}=0 \\
\left(\frac{\partial}{\partial t}+M \frac{\partial}{\partial x}\right) u+\frac{\partial p}{\partial x}=g_{1}(x, y, t), \\
\left(\frac{\partial}{\partial t}+M \frac{\partial}{\partial x}\right) v+\frac{\partial p}{\partial y}=g_{2}(x, y, t)
\end{gathered}
$$

where $g 1$ and $g 2$ model a Gaussian momentum source, which both oscillates sinusoidally and decays algebraically in time. Typical examples include, a quadrupole sound distribution. Here $g_{1}$ and $g_{2}$ are the gradient of a potential $\phi$ Such a function is given by

$$
\phi=A(t) e^{-\alpha R^{2}} \cos (2 \theta)
$$

where $\tan \theta=\frac{y-y_{0}}{x-x_{0}}, R=\sqrt{\left(x-x_{0}\right)^{2}+\left(y-y_{0}\right)^{2}},\left(x_{0}, y_{0}\right)$ is the location of the source, $\alpha$ is a positive constant, and $A(t)$ is the amplitude and a function of $t$. (In the numerical experiments $A=\sin 2 \pi t /\left(1+t^{2}\right)$ )

In a sample computation which was computed for a time length of 100 periods (22415 time steps), the solution obtained with the second order conditions was compared with the exact solution, a solution obtained by setting incoming characteristic variables to zero, and one obtained using the first order condition. The exact solution was computed in a large domain in which, within the tıme of computations, the waves could not reflect off the artificial boundaries and return to the small domain The maximum error in pressure calculations observed for the characteristic conditions was $10.3 \%$, for the first order asymptotic condition it was $3.3 \%$, and 
for the second order condition the error was $1.3 \%$; indicating the expected improvement. In Figures 5.1 and 5.2, the exact solution for the pressure 1s given after 5 periods and 10 periods of time respectively. Subsequent parrs of figures (5 3-5.4, 5 5-5 6 and 5 7-5.8) indicate the solution at these times for the characteristic boundary condition, the first order asymptotic condition, and the second order asymptotic conditions respectively At 10 periods the errors are visible in the first two cases and their orders of the magnitude indeed are as indicated above. Clearly the higher order condition improved the results 


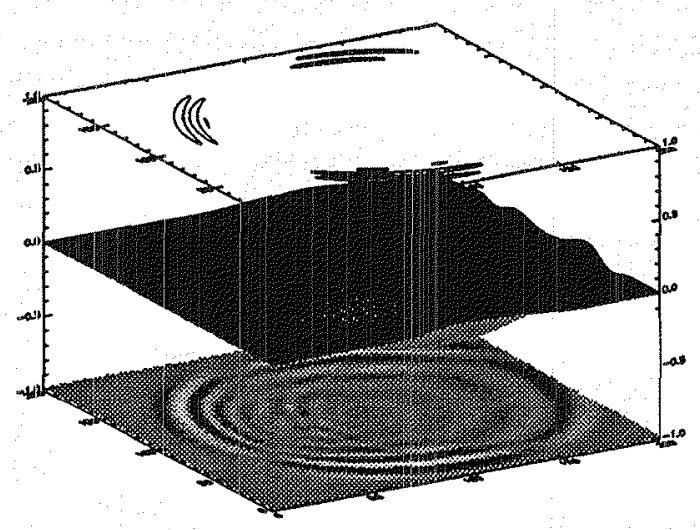

FIG. 5.1. Exact Solution at 5 periods of time

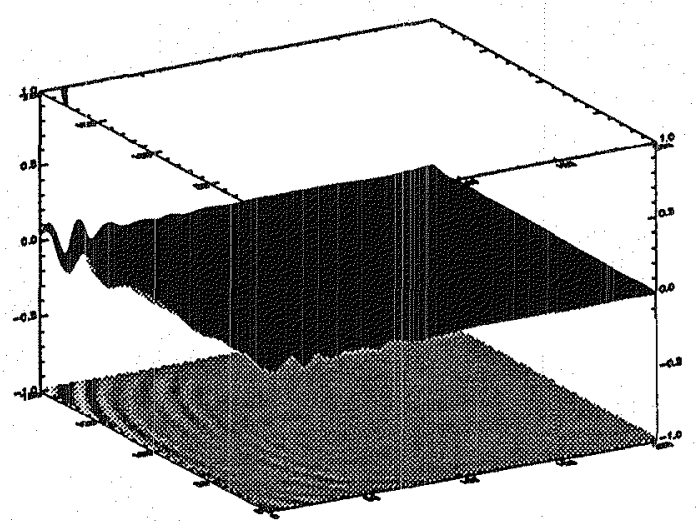

FiG. 5.2. Exact Solution at 10 periods of time 


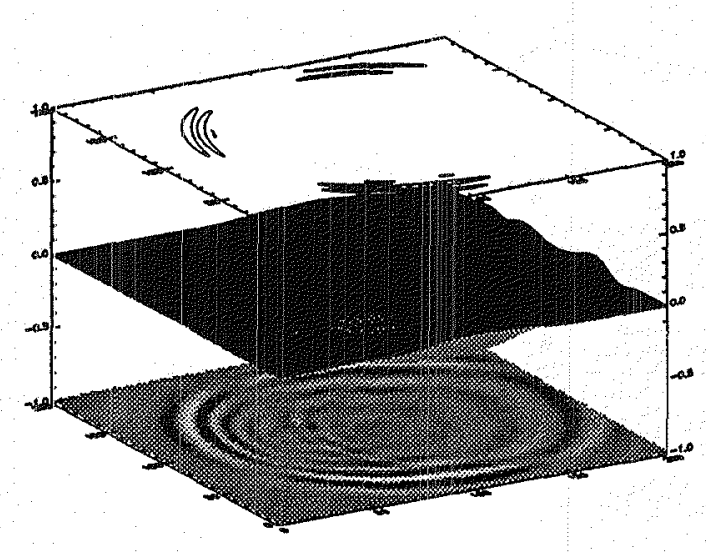

FIG. 5.3. Solution with characteristics based boundary condition $t=5$ periods

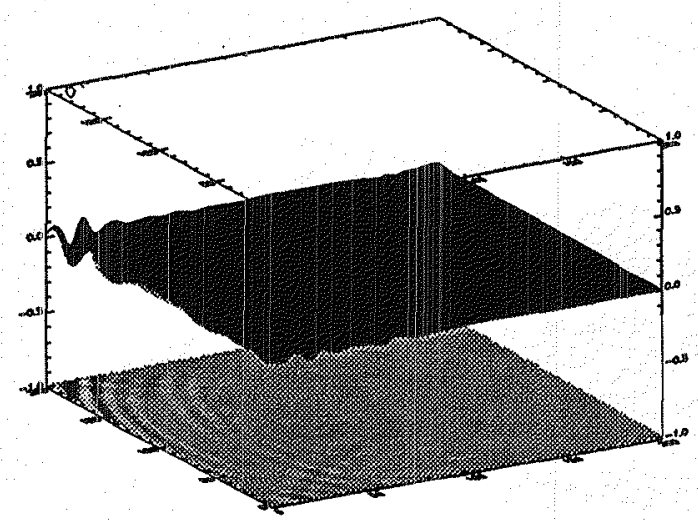

FIG. 5.4. Solution with characteristics based boundary condition $t=10$ periods 


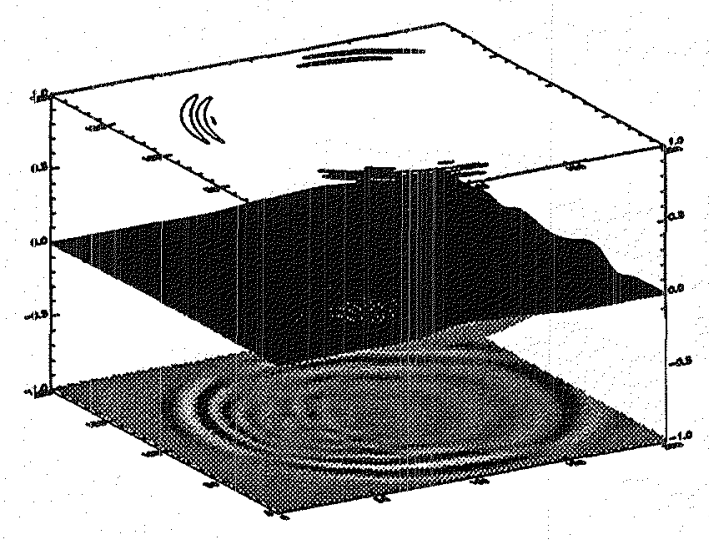

FIG. 5.5. Solution with first asymptotic boundary condition $t=5$ periods

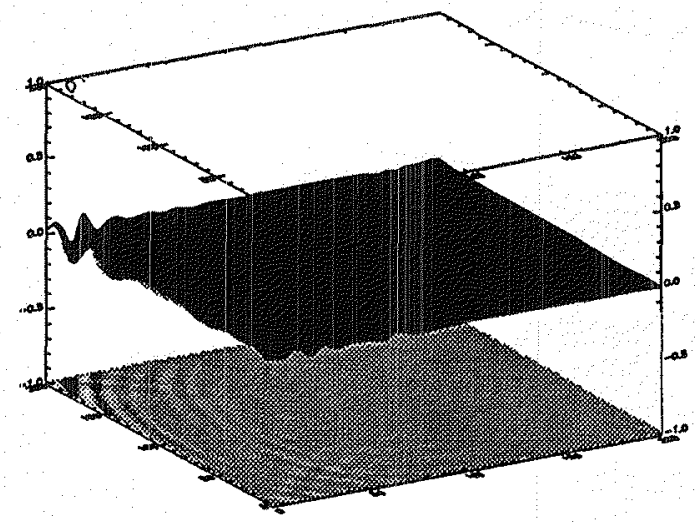

FIG. 5.6. Solution with first asymptotic boundary condition $t=10$ periods 


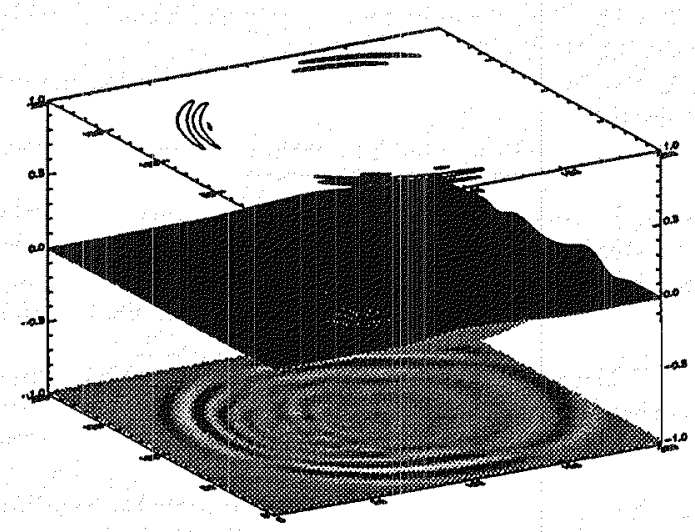

FIG. 5.7. Solution with second asymptotic boundary condition $t=5$ periods

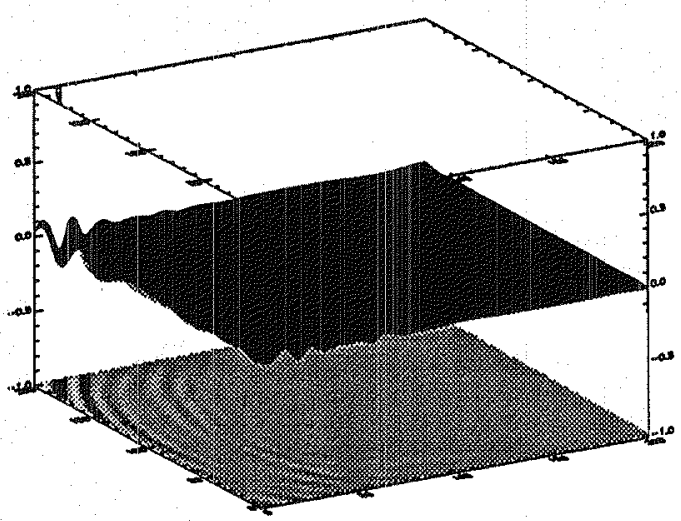

FIG. 5.8. Solution with second asymptotic boundary condition $t=10$ periods 


\section{REFERENCES}

[1] A Bayliss and E Turkel, "Radiation boundary conditions for wave hke equations", Commun Pure Appl Math, vol 23, (1980) pp 707-725

[2] A Bayliss and E Turkel, "Far field boundary conditions for compressible flows", J Comp Phys , 48, (1982), 182-199

[3] B Engquist and A Majda, "Absorbing boundary conditions for the numencal simulation of waves", Math Comp, 31, (1977), pp 629-651

[4] F G Fnedlander, "On the radiation field of pulse solutions of the wave equat1on", Proc Roy Soc A, 279, (1964), pp 386-394

[5] T Hagstrom, "On high-order radiation boundary conditions", this volume

[6] T Hagstrom, S I Hariharan, R C MacCamy, "On the accurate long-tıme solution of the wave equation in extenor domains Asymptotic expansions and corrected boundary conditions", Math Comp , Vol 63, No 208, (1994) pp 507-539

[7] S I Harzharan and T Hagstrom, "Far field expansion for anisotropic wave equations," Proceedings of the Second IMACS Symposrum on Computational Acoustics, North Holland, Eds D Lee, A Cakmak and R. Vichnevetsky, (1990), pp 283-294

[8] G A Knegsmann and C S Morawetz, "Solvng the Helmholtz equation for extenor problems with variable index of refraction $I^{n}$, SIAM J Stat Comput, Vol 1, No 3, (1980), pp 371-385

[9] G A Knegsmann, A Tafiove, and $K R$ Umashakar, "A new formulation of electromagnetic wave scattering using an on-surface radiation boundary condition approach", IEEE Trans Antennas Propagat, vol AP-35, no 2, pp 153-161, 1987 


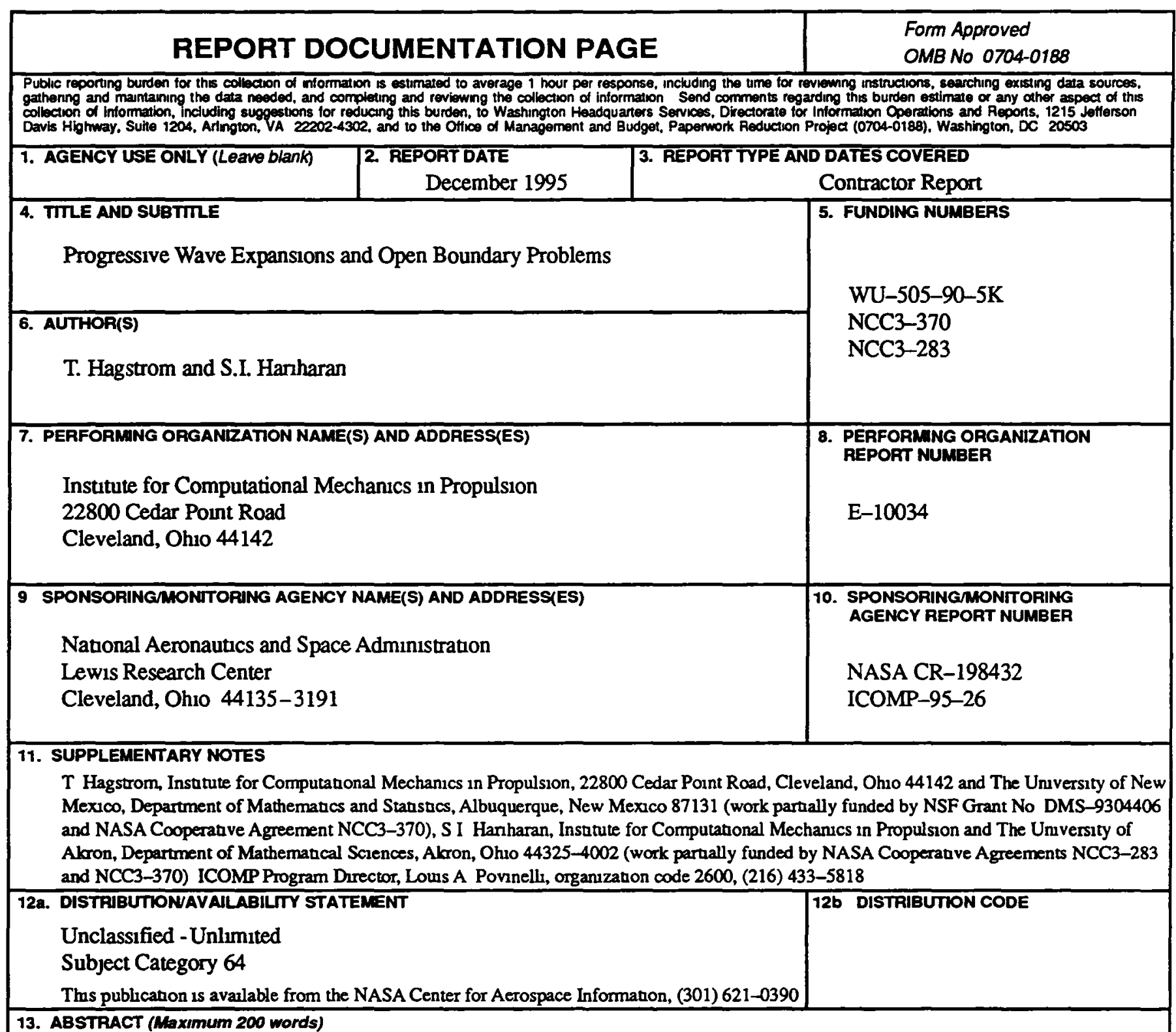

13. ABSTRACT (Maximum 200 words)

In this paper we construct progressive wave expansions and asymptotic boundary conditions for wave-like equations in exterior domains, including applications to electromagnetics, compressible flows and aero-acoustics. The development of the conditions will be discussed in two parts The first part will include derivations of asymptotic conditions based on the well-known progressive wave expansions for the two-dimensional wave equations A key feature in the denvations is that the resultung family of boundary conditions involve a single denvative in the direction normal to the open boundary These conditions are easy to implement and an application in electromagnetics will be presented. The second part of the paper will discuss the theory for hyperbolic systems in two dimensions Here, the focus will be to obtain the expansions in a general way and to use them to derive a class of boundary conditions that involve only tume denvatives or tume and tangential denvatives. Maxwell's equations and the compressible Euler equations are used as examples Simulations with the linearized Euler equations are presented to validate the theory

14. SUBJECT TERMS

Boundary conditions; Maxwell's equations; Euler equations; Numencal simulations 15 NUMBER OF PAGES

\begin{tabular}{|c|}
24 \\
16. PRICE CODE \\
A03 \\
\hline 20 LIMTTATION OF ABSTRACT \\
\hline
\end{tabular}

17. SECURTY CLASSIFICATION OF REPORT Unclassified

18. SECURTY CLASSIFICATION
OF THIS PAGE
Unclassified

Unclassified

SECURITY CLASSIFICATION
OF ABSTRACT
Unclassified


End of Document 